\title{
CONSTRUCCIONES DE PARTICIPIO Y DE INFINITIVO EN TEXTOS CATALANES (SIGLOS XIII-XV) ${ }^{1}$
}

\section{Introducción}

El objetivo de este estudio es examinar el orden de palabras en las construcciones constituidas por un verbo regente y un participio o un infinitivo dependiente en textos catalanes medievales y prerrenacentistas, con atención particular a la anteposición de las formas verbales dependientes y la separabilidad de los dos verbos que componen cada construcción. Dichos fenómenos fueron examinados en las construcciones de participio del español antiguo por Company ${ }^{2}$, Andres-Suárez ${ }^{3}$ y García Martín ${ }^{4}$, y en las construcciones de infinitivo del español y del portugués por Berta ${ }^{5}$. Los estudios dedicados a cuestiones de la sintaxis histórica del catalán como las gramáticas históricas clásicas de Badia i Margarit ${ }^{6}$ y de Moll $^{7}$ se preocupan más por los procesos que afectan la auxiliaridad de los verbos regentes de las construcciones perifrásticas y la concordancia del participio con el objeto directo, mientras que Nagy, en su artículo concentrado en la construcción anar+infinitiu ofrece una aproximación pragmática al proceso de gramaticalización de la construcción ${ }^{8}$. El orden de los constituyentes no parece interesar a los investigadores, a pesar de las diferencias que se observan entre la situación medieval y la actual y los paralelos que existen entre los dos tipos de construcción. En este trabajo nos proponemos contribuir al estudio de la evolución del orden de palabras de las construcciones en cuestión llamando la atención sobre la importancia de estudiar los fenómenos mencionados en ambos tipos de construcción en conjunto.

\footnotetext{
1 Agradezco a Diana Krekovics y Katalin Nagy la ayuda que me brindaron durante las investigaciones en la recogida de datos y la clasificación de las estructuras registradas.

${ }^{2}$ Concepción COMPANY, "Sintaxis y valores de los tiempos compuestos en el español medieval”, in: Nueva Revista de Filología Hispánica, XXXII/2, 1983, págs. 235-257.

${ }^{3}$ Irene ANDRES-SUÁREZ, El verbo español. Sistemas medievales y sistema clásico. Madrid, Gredos, 1994.

${ }^{4}$ José María GARCÍA MARTÍN, La formación de los tiempos compuestos del verbo en español medieval y clásico. Aspectos fonológicos, morfológicos y sintácticos. Valencia, Universitat de València, 2001.

5 Tibor BERTA, "La estructura de las construcciones de infinitivo en textos españoles y portugueses medievales”, in: Sándor KISS- Giampaolo SALVI-Ildikó SZIJJ (eds.), rom.hu. Études Romanes de Budapest, 3. Budapest, Programme Doctoral de Linguistique Romane de l'Université Eötvös Loránd/Íbisz, 2001, págs. 33-47. Idem, Clíticos e infinitivo. Contribución a la historia de la promoción de clíticos en español y portugués. Szeged, Hispánia, 2003.

${ }^{6}$ Antoni BADIA I MARGARIT, Gramàtica històrica catalana, València, Tres i Quatre, 1994.

${ }^{7}$ Francesc B. de MOLL, Gramàtica històrica catalana, València, Servei de Publicacions de la Universitat de València, 1991.

${ }^{8}$ Katalin NAGY, "Contribución a la historia de las perífrasis ir+a+infinitivo y anar+infinitiu. Una aproximación pragmática", in: Ádám ANDERLE (red.), Acta Universitatis Szegediensis: Acta Hispanica IX, Szeged, Universidad de Szeged, 2004, págs. 81-92.
} 
Desde el punto de vista del orden de los elementos constituyentes de las construcciones verbales el catalán presenta rasgos parecidos a los de las demás lenguas romances de la Península Ibérica. Así, mientras que en el catalán actual no es normal la anteposición del participio o del infinitivo al verbo regente flexionado, en el catalán medieval, de modo parecido a lo que se ha observado en el español y en el portugués medievales, tal orden era absolutamente normal; cf. los ejemplos del catalán actual que se aducen en $(1)^{9}$ y los ejemplos medievales que se presentan en (2).
a. $\quad$ *Llegit el meu germà ha el llibre.
b. $\quad$ *Llegir el llibre el meu germà va.
c. ?Llegir el llibre el meu germà pot.
d. ?Llegir el llibre el meu germà vol.
a. que tornada han la dita roba a una fembra públicha. (Lleida, 23, 79-80)
b. $\quad$ E açò que fer-na devem (Desclot, 15)
c. tota honor que fer pogueren al conte (ReiHon, 67)
d. e si fermar no volen (CriVal, 14r, R234)

Todas las frases que se presentan en (1) coinciden en no ser habituales en el catalán actual, aunque su aceptabilidad no es juzgada de la misma manera. (1a-b) se consideran claramente imposibles, es decir, son agramaticales en la actualidad. (1c-d), sin embargo, son posibles como enunciados enfáticos, aunque el orden con la anteposición "no es normal". La diferencia probablemente radica en el carácter auxiliar del verbo regente. En (1a-b) ha y va son formas verbales completamente desemantizados que desempeñan función únicamente gramatical, los cuales forman una unidad verbal con el participio y el infinitivo, respectivamente, que funciona como un único verbo. Entre estas circunstancias el orden de los verbos es rígidamente fijo: el verbo regente -auxiliar- precede inmediatamente al participio y al infinitivo. En (1c-d) las formas verbales pot y vol no tienen estos rasgos característicos, no son completamente desemantizados; dentro del complejo verbal el infinitivo es su complemento y ellos mismos funcionan como núcleo.

Los ejemplos medievales aducidos en (2) demuestran que la anteposición se documenta en los textos antiguos y podemos observar que este orden era normal prácticamente con cualquier verbo regente.

También se puede afirmar que mientras que en el catalán actual es prácticamente obligatoria la adyacencia léxica del verbo regente y del participio o del infinitivo dependiente, el catalán antiguo permitía el fenómeno de la interpolación, es decir, que cualquier contituyente se colocase entre los dos verbos. Cf. los ejemplos modernos que se aducen en (3) y los ejemplos medievales que se presentan en (4).
a. $\quad *$ El meu germà ha el llibre llegit.
b. $\quad *$ Ha el meu germà llegit el llibre.
c. *El meu germà ha tots els dies llegit el llibre.

\footnotetext{
${ }^{9}$ Agradezco la ayuda de la Dra. Sandra Montserrat en juzgar la gramaticalidad de las frases del catalán actual que se aducen en (1) y (3).
} 
d. *El meu germà va el llibre llegir.

e. $\quad *$ Va el meu germà llegir el llibre.

f. $\quad * E l$ meu germà va tots els dies llegir un llibre.

g. $\quad *$ El meu germà vol el llibre llegir.

h. ?Vol el meu germà llegir el llibre.

i. $\quad$ El meu germà vol tots els dies llegir un llibre.

j. $\quad * E l$ meu germà pot el llibre llegir.

k. ?Pot el meu germà llegir el llibre.

1. El meu germà pot tots els dies llegir un llibre.

(4) a. e agra a mi mort o nafrat si (CriVal, 7r, R224)

b. $\quad$ avets vosaltres fet so que les letres dien (ReiHon, 75)

c. lo havien molt fortment enclinat son pensament en aquestes coses terrenals. (AveMar, 118, 9-12)

d. ans que'ls francesos nos tenguen de totes Parts asetjats; (Desclot, 15)

e. $\quad$ con era aqui venguda (ReiHon, 72)

f. diens que no y podien àls fer que (CriVal, 26r, R250)

g. que ja hi puxa negú star a prou (Desclot, 44)

h. $\quad$ començaren cada dia de perseverar en lo peccat, (Lleida, 103, 9-10)

Los ejemplos modernos aducidos en (3a-f) demuestran que en las construcciones del perfecto compuesto con haver y del perfecto perifrástico con anar los dos verbos deben ser obligatoriamente adyacentes y no pueden ser separados por ningún elemento intercalado entre ellos, es decir, la interpolación es agramatical. En las frases de (3g-1), en las que los verbos regentes son voler y poder, la situación es más complicada. Mientras que la interpolación del argumento interno -objeto directo- en $(3 \mathrm{~g})$ y (3j) se siente claramente agramatical, la del sujeto en (3h) y (3k), aunque no es habitual es posible en casos de énfasis. La intercalación del complemento circunstancial ilustrada en (3i) y (31) se tiene por mucho más habitual en las construcciones de infinitivo, y puede ser considerada gramatical.

Los ejemplos medievales presentados en (4) ilustran la interpolación que se observa en los textos antiguos. Se puede comprobar que en ellos se documenta la interpolación de cualquier constituyente tanto en las construcciones de participio -(4a-e)- como en las construcciones de infinitivo -(4f-h)-: (4a) ilustra la interpolación del argumento interno -objeto directo- y (4b) la del sujeto en la construcción de participio con haver; (4c-e) ilustran la interpolación del complemento circunstancial en construcciones de participio con diversos verbos regentes; (4f) ilustra la intercalación del argumento interno -objeto directo-, (4g) la del sujeto y (4h) la del complemento circunstancial en construcciones de infinitivo con diversos verbos regentes.

Incluso sin entrar en un análisis pormenorizado se puede concluir que, a diferencia de la situación que se observa en el catalán actual, que limita interpolación de constituyentes tanto en las construcciones de participio como en las construcciones de infinitivo, en el catalán medieval ambas construcciones aceptaban la intercalación de cualquier constituyente entre sus componentes verbales.

Más arriba ya nos hemos ocupado de las causas estructurales responsables de las diferencias que se notan entre el estado medieval y el actual en relación a la anteposición del participio y del infinitivo dependientes y a la interpolación. Aquí 
sólo nos referimos a los elementos más importantes del marco teórico de las explicaciones. La anteposición de los verbos complementos, así como la interpolación eran posibles en la variante medieval de todas las lenguas romances peninsulares gracias al funcionamiento del sistema V2, que colocaba el verbo en la segunda posición de la frase y permitía que cualquier constituyente ocupase la primera. La sustitución de este sistema por el nuevo orden SVO causaría que el participio y el infinitivo -como complemento regido por el verbo regente- se situase a la derecha de éste y que los demás complementos desapareciesen de las posiciones intermedias. La consecuencia de estos cambios sería, pues, que se fijasen como órdenes habituales $\operatorname{VPart}(\mathrm{O})$ y $\operatorname{VInf}(\mathrm{O})$ en la variedad actual del catalán ${ }^{10}$.

Actualmente tenemos suficientes conocimientos acerca de la naturaleza de los cambios lingüísticos para pensar que la anteposición y la interpolación, rasgos característicos de la lengua medieval, no habrán desaparecido de repente, sino gradualmente en un período de transición entre la fase medieval y la fase actual de la historia de la lengua. También es de suponer, aunque no se dispone de referencias tan concretas como en el caso del español al respecto, que las estructuras antiguas pueden surgir en el catalán actual, sea por arcaísmo de algún autor sea por las características de algún tipo de texto. Incluso es lógico conjeturar -como hicimos durante el análisis de los mismos fenómenos en español y portugués- que la anteposición y la interpolación, que en la Edad Media eran solamente alternativas de otras posibilidades sintácticas, originariamente no se usarían con la misma frecuencia en los distintos tipos de textos, y que su retroceso tampoco ocurriría necesariamente con la misma rapidez en los diferentes géneros o estilos literarios. Para comprobar la validez de esta hipótesis se realizó el análisis de la frecuencia estadística de la anteposición del participio y del infinitivo en textos catalanes medievales procedentes del período que abarca los siglos entre XIII y XV. Los objetivos eran idénticos a los que se habían formulado en las investigaciones referentes al español y al portugués:

a) comparación de las construcciones de participio con las de infinitivo;

b) comparación sincrónica de diferentes tipos de textos de la misma época;

c) comparación de textos del mismo tipo procedentes de épocas distintas.

Para alcanzar estos objetivos se creó un corpus constituido por textos catalanes redactados entre el siglo XIII y el siglo $\mathrm{XV}$, que pertenecen a los siguientes tipos: textos jurídicos, crónicas y obras literarias. Se ha excluido la poesía para evitar la influencia del ritmo, de la versificación y las necesidades de rima que pueden permitir estructuras que no se sienten normales. El Cuadro 4.1. presenta los datos más importantes del corpus seleccionado ${ }^{11}$. En la columna "Palabras" aparece la extensión

${ }^{10} \mathrm{~V}$ equivale a verbo regente, Part a participio, Inf a infinitivo y $(O)$ representa la posición de los complementos opcionales en la frase.

${ }^{11}$ Una parte de los textos catalanes analizados procede del Corpus Informatitzat del Català Antic (CICA), J. Torruella (dir.). http//seneca.uab.es/sfi/cica. 2006. Estos textos son los siguientes: Lleida=Processos de crims del segle XV a Lleida: Transcripció i estudi lingüístic, a cura de Maria Dolors FARRENY I SISTAC, Lleida, Institut d'Estudis Ilerdencs, 1986, 21-49. [siglo XV]; Desclot=Bernat DESCLOT, Crònica, vol. V, Barcelona, Barcino, «Els Nostres Clàssics», 1951 [siglos XIII-XIV]. Agradezco a la Dra. Sandra Montserrat (Universitat d'Alacant) que haya puesto a mi disposición la versión electrónica de estos dos textos. Los demás textos analizados en versión impresa son los siguientes: AveMar=Ramon Llull, Llibre d'Ave Maria, in: Ramon LLULL, Llibre d'amic e amat. Llibre d'Ave Maria. Barcelona, Ed. 
de cada texto o fragmento examinado; en la columna "Inf" figura el número de aquellas construcciones registradas que contienen un verbo regente y un infinitivo dependiente; las cifras bajo "Part" se refieren a las construcciones de participio que contienen un verbo regente cuyo complemento es un participio; la columna "Total" indica el número de todos los ejemplos registrados en un texto analizado. Nótese que se han excluido los participios absolutos y las construcciones con infinitivo de función adverbial, porque en estos casos el participio y el infinitivo no dependen estrictamente del verbo flexionado.

\begin{tabular}{|l|r|r|r|r|}
\hline \multicolumn{1}{|c|}{ Textos catalanes } & \multicolumn{1}{c|}{ Palabras } & \multicolumn{1}{c|}{ Inf } & \multicolumn{1}{c|}{ Part } & \multicolumn{1}{c|}{ Total } \\
\hline AveMar & 9.000 & 100 & 72 & 172 \\
\hline ReiHon & 8.000 & 154 & 159 & 313 \\
\hline Desclot & 10.000 & 143 & 176 & 319 \\
\hline CriVal & 10.000 & 112 & 301 & 413 \\
\hline Vesp & 13.000 & 311 & 97 & 408 \\
\hline Lleida & 7.800 & 368 & 395 & 763 \\
\hline Total catalanes & 57.800 & 1.188 & 1.200 & 2.388 \\
\hline
\end{tabular}

Cuadro 4.1.

Extensión del corpus catalán analizado

A continuación se presentarán los casos de anteposición e interpolación recogidos durante las investigaciones y se analizarán brevemente los datos de frecuencia de ambos fenómenos en los textos seleccionados.

\section{Proporción de la anteposición}

El primer fenómeno analizado ha sido el de la anteposición del verbo en forma de participio o infinitivo, ya ilustrado en los estudios precedentes de este tomo. Tal fenómeno se documenta también en los textos catalanes analizados con ambos tipos de construcciones. El Cuadro 4.2. resume los datos referentes a la proporción de la anteposición del participio al verbo regente, el cual puede ser haver, ésser o bien tenir.

Barcino, 1985. Reimpresión de la edición de 1927 [siglos XIII-XIV]; CriVal=Clams $i$ crims en la València medieval segons el Llibre de cort de Justícia (1279-1321), a cura de Maria Àngels DIÉGUEZ SEGUÍ, Alacant, Universitat d'Alacant, Departament de Filologia Catalana, 2002, 113-147 [1287]; ReiHon=Historia del rey de Hungría, en P. de BOFARULL I MASCARÓ (ed.), Colección de documentos inéditos del Archivo General de la Corona de Aragón. Tomo XIII, Documentos literarios en antigua lengua catalana, Barcelona, Imprenta del Archivo, 1857. Reimprimido en 1973 por Imp. Vda. Fidel Bot. [siglo XIV]; Vesp=Sitio y destrucción de Jerusalén por el emperador Vespasiano en P. de BOFARULL I MASCARÓ (ed.), Colección de documentos inéditos del Archivo General de la Corona de Aragón. Tomo XIII, Documentos literarios en antigua lengua catalana. Barcelona, Imprenta del Archivo, 1857. Reimprimido en 1973 por Imp. Vda. Fidel Bot. [siglo XV]. Nótese que por razones tipográficas en los ejemplos publicados en este estudio algunas grafías de las ediciones mencionadas pueden haber sido modificadas. 


\begin{tabular}{|l|r|r|r|}
\hline \multicolumn{1}{|c|}{ Textos } & \multicolumn{1}{|c|}{ VPart } & \multicolumn{1}{c|}{ PartV } & \multicolumn{1}{c|}{ Total } \\
\hline AveMar & 67 & 5 & 72 \\
\hline Desclot & 169 & 7 & 176 \\
\hline CriVal & 285 & 16 & 301 \\
\hline ReiHon & 154 & 5 & 159 \\
\hline Vesp & 94 & 3 & 97 \\
\hline Lleida & 381 & 14 & 395 \\
\hline Total & 1.150 & 50 & 1.200 \\
\hline \multicolumn{4}{|c}{ Cuadro 42} \\
\end{tabular}

Anteposición del participio en textos catalanes

Se puede ver que la anteposición del participio al verbo regente ocurre 50 veces en un total de 1200 casos de construcciones de participio. Esta cifra constituye el $4 \%$ de todos los ejemplos recogidos con participio. Los casos con participio antepuesto se presentan a continuación.

(5) a. Ni com benauirats són aquells qui (AveMar, 112, 29-30)

b. Vençut és mon poder (AveMar, 113, 16-17)

c. exalçat és l'honrament de Nostra Dona (AveMar, 113, 17-18)

d. Consolat, alegrat me té Nostra Dona (AveMar, 113, 23) (1)

e. lo pagès dix que vençut e sobrat l'havia Dominus tecum (AveMar, $124,30-31-125,1)$

(6) a. plorant de piatat que havia del rey e de les paraules que dites li havia. (Desclot, 7)

b. molt só pagat de vós e de la resposta que feta m'avets (Desclot, 8)

c. que nós levarem d'assò nostra deuta que dit havets (Desclot, 13)

d. que en les paraules que dites havia mostrave gran fe e gran amor al senyor rey (Desclot, 15)

e. que fes en aquella manera que dit havia. (Desclot, 16)

f. jo-us grahesch molt les paraules que dites havets (Desclot, 32)

g. que ja li valgués guiatge ne asegurament que fet li hagués, (Desclot, 45)

(7) a. segons que posat és (CriVal, 1v, R217)

b. $\quad$ si vedat no li fos (CriVal, 7r, R224)

c. $\quad$ segons que dit és (CriVal, 18r, R237)

d. en aitant com posades són contra él (CriVal, 23r, R247)

e. qui preses són (CriVal, 23r, R246)

f. pus que nafrat era (CriVal, 23r, R247)

g. $\quad$ e no per mal que feit aguessen él ni son frare (CriVal, 23r, R247)

h. en aitant com posades són contra él (CriVal, 23v, R248)

i. cridaren contra él per rahon del dit ahuyr que feit avia (CriVal, 23v, R248)

j. $\quad$ mort só, da'm de l'aygua (CriVal, 23v, R248)

k. que morts sots (CriVal, 25v, R249)

1. que forçats eren (CriVal, 25v, R249) 
m. segons que dit és (CriVal, 29r, R253)

n. segons que acostumat és d'aplegar (CriVal, 29r, R253)

o. en aitant com posades són contra él (CriVal, 45v, R255)

p. dix que comprada la avia d'un corredor per IIII sols meis I diner (CriVal, 56r, R257)

(8) a. la regina qui morta es (ReiHon, 54)

b. la regina qui morta es (ReiHon, 54bis)

c. la regina qui morta es (ReiHon, 55)

d. per lo sagrament que fet avets (ReiHon, 58)

e. per lo sagrament que fet avets (ReiHon, 65)

(9) a. un temple lo pus bel que hanc bastit fos (Vesp, 10)

b. qui creura lo fil de la Verge e sera batayat salvat sera (Vesp, 14)

c. Jacob lo savi juheu trames a I seu misatge (Vesp, 15)

(10) a. que hoyt ha dir (Lleida, 23, 79)

b. $\quad$ que tornada han la dita roba a una fembra públicha. (Lleida, 23, 79-80)

c. dix que hoc, dit-lo y havien (Lleida, 26, 44)

d. $\quad$ segons dit ha lo dit Nicholau (Lleida, 31, 53-4)

e. ço que dit ha (Lleida, 43, 122)

f. $\quad$ ço que dit li havia (Lleida, 48, 106)

g. de les que dites ha (Lleida, 73, 27)

h. $\quad$ ni de res que vist haguessen. (Lleida, 84, 24-5)

i. $\quad$ dit $\mathrm{m}$ ' an que (Lleida, 90, 24)

j. $\quad$ com dit ha (Lleida, 91, 19-20)

k. com los dessús dits instruïda la avien (Lleida, 97, 20)

1. que ffet havie (Lleida, 97, 24)

m. que ffet havie (Lleida, 97, 26)

n. que ffetes havie (Lleida, 99, 40)

La anteposición del verbo no flexionado se docuementa también en las construcciones de infinitivo de todos los textos catalanes analizados. El Cuadro 4.3. presenta la proporción de este fenómeno en este tipo de construcciones.

\begin{tabular}{|l|r|r|r|}
\hline \multicolumn{1}{|c|}{ Textos } & \multicolumn{1}{c|}{ VInf } & \multicolumn{1}{c|}{ InfV } & \multicolumn{1}{c|}{ Total } \\
\hline AveMar & 93 & 7 & 100 \\
\hline Desclot & 139 & 4 & 143 \\
\hline CriVal & 109 & 3 & 112 \\
\hline ReiHon & 153 & 1 & 154 \\
\hline Vesp & 306 & 5 & 311 \\
\hline Lleida & 365 & 3 & 368 \\
\hline \multicolumn{1}{|c|}{ Total } & 1.165 & 23 & 1.188 \\
\hline \multicolumn{4}{|c|}{ Cuadro 4.3. } \\
\hline
\end{tabular}

Anteposición del infinitivo en textos catalanes 
Los datos del Cuadro 4.3 indican que la anteposición del infinitivo ocurre 23 veces en el corpus examinado. Esta cifra es algo inferior al 2\% del total de las 1188 construcciones de infinitivo. Los casos recogidos se presentan en (11-15).

(11) a. Si fer se podia (AveMar, 113, 20)

b. $\quad$ ans que parlar se poguessen (AveMar, 120, 24-25)

c. Mas cogità l'abat que a davallar lo convenia (AveMar, 123, 17)

d. A plorar me convé, e a fer penitència (AveMar, 112, 8-9)

e. e a sostenir me convé aspra vida (AveMar, 112,9)

f. e a lloar, amar, conèixer, servir me convé tu (AveMar, 112, 9-10)

g. A plorar me convé la mort dels pobres qui morran (AveMar, 115, 16-17)

(12) a. E açò que fer-na devem (Desclot, 15)

b. aplegaren aquella roba que portar -se'n pogueren. (Desclot, 17)

c. si no trobats los $\mathrm{h}(\mathrm{o})$ mens qui romanir hich $\mathrm{v}(\mathrm{u})$ llan (Desclot, 30)

d. $\quad$ si fer no.(h)u volia (Desclot, 42)

tota honor que fer pogueren al conte (ReiHon, 67)

(14) a. e si fermar no volen (CriVal, 14r, R234)

b. $\quad$ ni noure porie (CriVal, 18r, R237)

c. perquè en veritat per les dites rahons provar no-s poria ni profitaria al dit Jacme (CriVal, 18r, R238)

(15) a. ni gritar ni enviar nols hich pot hom per loync que (Vesp, 35)

b. negu qui batayar se vulla no li sia contrastat (Vesp, 21)

c. e cant veren que morir los convenia aqui de fam (Vesp, 24)

d. que aver la pogesen (Vesp, 36)

e. $\quad$ e aytentost com fumar hi veyen entravensen dintre (Vesp, 36)

f. $\quad$ si desolarte podien (Lleida, 85, 18)

g. donar-los-hi à a ella (Lleida, 103, 4-5)

h. hon ella anar avie (Lleida, 97, 25)

Al comparar la frecuencia de la anteposición del participio con la del infinitivo encontramos, pues, que el participio se antepone a su verbo regente dos veces más que el infinitivo. Si se contrastan los datos de las dos construcciones por textos, destaca que el único texto en el que el porcentaje de la anteposición es semejante en ambas construcciones es AveMar, con el 6,9\% con participio y con el $7 \%$ con infinitivo. En todos los demás casos es superior la frecuencia de la anteposición del participio a la del infinitivo: Desclot: $4 \%$ y 2,8\%; CriVal: $5,3 \%$ y 2,7\%; ReiHon: $3,1 \%$ y $0,6 \%$; Vesp: $9,3 \%$ y $1,6 \%$; Lleida: $3,5 \%$ y $0,8 \%$. Nótese, además, que los 5 casos de anteposición en ReiHon son, en realidad, repeticiones de sólo dos frases. 


\section{Intercalación de constituyentes en catalán}

En los textos catalanes analizados -así como en los textos españoles y portugueses examinados anteriormente (véanse los estudios correspondientes de este mismo tomo)se documenta la interpolación de diferentes constituyentes entre el verbo regente flexionado y el participio o el infinitivo dependiente. El Cuadro 4.4. presenta los datos referentes a la intercalación de constituyentes entre el verbo regente y el participio ${ }^{12}$.

\begin{tabular}{|c|c|c|c|c|c|c|}
\hline Textos & \multicolumn{4}{|c|}{ Int+ } & Int- & Total \\
\hline & Sujeto & Objeto & Adverbio & Int+ total & & \\
\hline AveMar & 0 & 0 & 4 & 4 & 68 & 72 \\
\hline Desclot & 3 & 6 & 12 & 21 & 155 & 176 \\
\hline CriVal & 1 & 2 & 9 & 12 & 289 & 301 \\
\hline ReiHon & 2 & 0 & 16 & 18 & 141 & 159 \\
\hline Vesp & 0 & 2 & 9 & 11 & 86 & 97 \\
\hline Lleida & 1 & 1 & 3 & 5 & 390 & 395 \\
\hline Total & 7 & 11 & 53 & 71 & 1.129 & 1.200 \\
\hline
\end{tabular}

Interpolación de constituyentes con participio en textos catalanes

En los textos analizados se han recogido en total 71 construcciones en las que el verbo regente -por lo general haver o ésser- está separado del participio dependiente que lo sigue. Esta cantidad de ocurrencias constituye el 5,9\% de las 1.200 construcciones de participio.

Si analizamos los datos de cada uno de los textos del corpus, encontramos datos bastante diferentes. La proporción de la interpolación de constituyentes alcanza los siguientes porcentajes en los textos: AveMar: 0,6\%, Desclot: 11,9\%, CriVal: 3,9\%, ReiHon: $11,3 \%$, Vesp: 11,3\%, Lleida: $1,3 \%$. Los datos indican frecuencia baja que oscila entre el $0,6 \%$ y el 3,9\% en tres textos -AveMar, CriVal y Lleida-, dos de los cuales son textos de carácter jurídico. En los otros tres textos la frecuencia de la interpolación es más alta y más estable, ya que se encuentra entre el 11 y el $12 \%$. Todos estos textos -Desclot, ReiHon y Vesp- son de carácter más o menos literario, narrativo.

Según la categoría del constituyente interpolado destaca la proporción de los elementos adverbiales: éstos aparecen entre los dos verbos 53 veces, es decir, en el $4,4 \%$ del total de las construcciones recogidas. El sujeto se interpola 7 veces $0,6 \%$, mientras que algún argumento regido 11 veces, o sea en el 9,2\% de los 1.200 casos. Los casos de interpolación con elementos adverbiales se presentan en (16-20), los casos con sujeto interpolado en (21-24), mientras que los casos con argumento regido en (25-27).

\footnotetext{
${ }^{12}$ Así como en los demás estudios de este tomo las abreviaturas de los cuadros referentes a la interpolación tienen los significados siguientes: Int+=con interpolación; Int-=sin interpolación; Sujeto=sujeto interpolado; Objeto=complementos regidos interpolados; Adverbio=elementos adverbiales interpolados.
} 
(16) a. cor del blat havia tan poc atrobat en les granges que (AveMar, 114, 30-31)

b. ni con és bellament il-luminat per lo sol (AveMar, 113, 2)

c. cor aquell qui li havia tramès era tot despès (AveMar, 116, 23-24)

d. cor los grans negocis temporals, los quals lo convenia tractar per lo monestir, lo havien molt fortment enclinat son pensament en aquestes coses terrenals. (AveMar, 118, 9-12)

(17) a. han ja combetut. (Desclot, 11)

b. noúus cuydets que yo hage en açò consentit; (Desclot, 7)

c. e a tan gran poder com ell ha ab si manat; (Desclot, 11)

d. car ell e lo rey de Mallorques havien ja parlat ab alguns hòmens de Castalló que (Desclot, 19)

e. ans éran dolents com lo rey e los cavallers los eran axí scapats, (Desclot, 26)

f. qui és assí legat (Desclot, 44)

g. que éran ab ells venguts de la terra del compta d' Empúrias (Desclot, 48)

h. $\quad$ cels qui éran ab ell I miller cavalcat (Desclot, 26)

i. $\quad$ que vós ho havets molt ben tocat. (Desclot, 7)

j. ans que $1 \mathrm{ls}$ francesos nos tenguen de totes Parts asetjats; (Desclot, 15)

k. que.l hach ben asejat (Desclot, 9)

1. que se'n foren tuyt anats si gossassen (Desclot, 33)

(17) a. e la mala fama sera tost passada (ReiHon, 55)

b. $\quad$ El infant era benn odrit (ReiHon, 64)

c. no fo anch mes hoit que (ReiHon, 56)

d. mos contes e mos barons man molt pregat que (ReiHon, 56)

e. Vos non serets axi tengut a Deu (ReiHon, 61)

f. que nos som fort despagats e hirats (ReiHon, 68)

g. E cant ageren molt sercat (ReiHon, 54)

h. E cant ach un petit estat (ReiHon, 60)

i. que sots axi anemorat (ReiHon, 60)

j. $\quad$ com era aqui venguda (ReiHon, 62)

k. con era aqui venguda (ReiHon, 72)

1. car per aventura lo comte es axi irat contra ella (ReiHon, 71)

m. quel comte ne sera fort despaguat (ReiHon, 71)

n. con nos a axi anganats (ReiHon, 69)

o. $\quad$ E cant la ach molt guardade (ReiHon, 77)

p. que hanc no les avie audes levat I. filet fort soptill (ReiHon, 73)

(18) a. que la dita na Guillemona li sia sentencialment condempnada (CriVal, 5r, R222)

b. que vo per vós dit justícia lo dit Berthomeu me sia per sentència condempnat per la dita força e violènçia e trencament d'alberch a mi feyt (CriVal, 7r, R224)

c. Deman per ço yo dit en Michel, en lo nom damunt dit, lo dit en Jafudà Alatzar ésser sentencialment condampnat en pagar a mi en 
nom del seynor rey los dua míllia sols deguts al dit David Mascaran (CriVal, 18v, R241)

d. que són públicament [acusats] d'omicidi (CriVal, 29r, R253)

e. On demane que.1 dit P. Cardona, corredor, li sie per vós séyner justícia e per vostra sentència condampnat per rahon del dit tradiment d'espaa (CriVal, 38r, R253b)

f. Hon demane lo dit Salamó que-l dit Johan Roso li sie sentencialment [condemnat per les] injúries a si feites (CriVal, 49v, R257)

g. $\quad$ que·l dit deute fo per lo dit Jafudà Alatzar degut (CriVal, 18, R241)

h. On demane la dita na Maria que la dita na Blanca li sie sentencialment condempnada per lla dita nafra e ferida a si feita (CriVal, 50r, R258)

(19) a. que io som asi vengut (Vesp, 13)

b. $\quad$ El sera tost guarit de la lebrosia (Vesp, 14)

c. que era fort destrouit de la lebrosia (Vesp, 15)

d. $\quad$ E havetsho mal fet (Vesp, 16)

e. e vos serets tentost guarit (Vesp, 18)

f. de la qual vos erets fort destret (Vesp, 21)

g. car vos sots ten be garit de vostra maleutia (Vesp, 22)

h. e cant ach longament preycat (Vesp, 20)

i. $\quad$ e cant agueren aqui refrescat (Vesp, 23)

(20) a. hi aveu ben ajudat! (Lleida, 70, 109-10)

b. $\quad$ que dissapte a vespre are prop passat (Lleida, 26, 50)

c. $\quad$ per quant dita sa mare havie molt parlat ab sa filla (Lleida, 81, 23-4)

(21) a. E foren-se tots guarnits ab lurs cavalls (Desclot, 18)

b. les paraules que vós havets dites a mi, he yo bé enteses; (Desclot, 7)

c. que a penes se fo lo dia sclarit (Desclot, 18)

que sien ambdosos pe[ng]ats (CriVal, 9r, R227)

(23) a. per totes les belleses qui son en vos ne son jo anamorat especialment per les belles mans (ReiHon, 58)

b. avets vosaltres fet so que les letres dien (ReiHon, 75)

(25) a. E axí com lo rey e los altres hagueren açò hoït (Desclot, 22)

b. quant lo rey, e lo compta e los altres cavallers hagueren açò entès (Desclot, 23)

c. quant los promens de les osts hagueren açò parlat ab lo rey (Desclot, 31)

d. que no hauran res acabat. (Desclot, 29)

e. $\quad$ que y hage res fet per flaquesa de cor (Desclot, 29)

f. no és null hom al món que d'aytal rahó me hagués res dit ne parlat, (Desclot, 45) 
(26) a. e agra a mi mort o nafrat si (CriVal, 7r, R224)

b. e deman que les dites quantitats de diners que a mi foren emblades sien a mi ajutgades i restituïdes dels béns dels dit malfeitors (CriVal, 47r, R256)

(27) a. Lemperador ac son navili aperalat entre naus lenys e galeas (Vesp, 23)

b. que io no tornare denant ell tro que aja consell trobat (Vesp, 14)

c. ell ja-u havie tot dit a dits stodiants. (Lleida, 86, 39-87, 1-2)

Los datos referentes a la interpolación de constituyentes entre el verbo regente y el infinitivo dependiente se presentan en el Cuadro 4.5.

\begin{tabular}{|c|c|c|c|c|c|c|}
\hline Textos & \multicolumn{4}{|c|}{ Int+ } & Int- & Total \\
\hline & Sujeto & Objeto & Adverbio & Int total & & \\
\hline AveMar & 1 & 1 & 7 & 9 & 91 & 100 \\
\hline Desclot & 3 & 3 & 15 & 21 & 122 & 143 \\
\hline CriVal & 0 & 21 & 3 & 24 & 88 & 112 \\
\hline ReiHon & 2 & 6 & 4 & 12 & 142 & 154 \\
\hline Vesp & 12 & 10 & 11 & 33 & 278 & 311 \\
\hline Lleida & 5 & 5 & 12 & 22 & 346 & 368 \\
\hline Total & 23 & 46 & 52 & 121 & 1.067 & 1.188 \\
\hline
\end{tabular}

Interpolación en construcciones de infinitivo

En los textos analizados se han recogido en total 121 construcciones de infinitivo en las que éste y el verbo regente están separados por algún otro constituyente oracional. Esta cifra de ocurrencias constituye el $10 \%$ de las 1.188 construcciones de infinitivo recogidas en total en todos los textos catalanes examinados.

El análisis de los datos correspondientes a cada uno de los textos ofrece los siguientes porcentajes: AveMar: 9\%, Desclot: 14,6\%, CriVal: 21,4\%, ReiHon: 7,9\%, Vesp: $10,6 \%$, Lleida: $6 \%$. Según estas cifras en tres textos -AveMar, ReiHon y Vespla proporción de la interpolación en las construcciones de infinitivo se coloca alrededor del 9\%. En los dos textos procedentes del siglo XV -Vesp y Lleida- el porcentaje difiere aunque no considerablemente de esta cifra. Sólo el porcentaje registrado en CriVal es sorpredentemente alto para nuestras expectativas, que se apoyan en los datos observados en textos españoles, portugueses y los demás textos catalanes. Tal anomalía, sin embargo, puede ser explicada si se tiene en cuenta que en este corpus, constituido por diferentes textos relativamente cortos, varias construcciones de infinitivo latinizantes se repiten monótonamente, por lo cual estas estructuras no tienen el mismo peso que las demás.

Por lo que se refiere a la frecuencia de interpolación de constituyentes de categoría diferente, podemos afirmar que ésta alcanza cifras diferentes con diferentes tipos de constituyentes intercalados.

El elemento que más veces se intercala entre el verbo regente y el infinitivo es el complemento circunstancial o algún elemento adverbial. Este tipo de interpolación se ha 
registrado 121 veces en los textos analizados (10,2\% del total de las construcciones de infinitivo). Los casos recogidos se detallan en los ejemplos que van del (28) al (33).

(28) a. que aquella almoina que l'abat faïa no podia llongament durar (AveMar, 114, 26-27)

b. per les quals pogués molt amar e honrar Nostra Dona (AveMar, 119, 28-29)

c. $\quad$ ab què et pusca plenament amar e conèixer (AveMar, 119, 13)

d. ab la qual la pogués complidament conèixer (AveMar, 120, 1-2)

e. Volria's per vós inclinar a pregar son Fill con ... (AveMar, 121, 9-10)

f. E per açò devem tuit fer reverència e honor a Dominus tecum (AveMar, 125, 29-30)

g. que anava sovint visitar lo monge qui (AveMar, 126, 20-21)

(29) a. adonchs no ho pot pus soferir cant ... (ReiHon, 63)

b. $\quad$ E jo am mes predre les mans que (ReiHon, 58)

c. E la contesa novela so es la muler del sabe ja perlar aquel lenguatge (ReiHon, 63)

d. $\quad$ E mantinent ela vee davant laltar penjar dues mans (ReiHon, 73)

(30) a. car vós no·us devets tant aventurar com nosaltres (Desclot, 12)

b. qui·ls viren axí axir spertament (Desclot, 17)

c. e feren semblant que daguessen aquí aturar lonch temps. (Desclot, 17)

d. que vullats per mi ne per vostra senyor lo compta soferir tant de mal (Desclot, 20)

e. majorment que me fe ne pogués menys valer. (Desclot, 22)

f. troba'l que.s volia ja colguar (Desclot, 23)

g. E lo rey féu mantinent rapicar los senys (Desclot, 24)

h. e feya defora al so axir tots los hòmens de la vila stadants (Desclot, 24)

i. $\quad$ qui·ls hoý axí plorar darrera ell (Desclot, 26)

j. $\quad$ que si nós los ne podiem vuy gitar ab honrament (Desclot, 29)

k. que no·s podían més tenir, (Desclot, 39-40)

1. e axí no·ls calia sinó pendra (Desclot, 39)

m. Lo cardenal féu allà coronar a Carlot, fill dell rey de Franssa, (Desclot, 40)

n. meseren-sa mans a fugir (Desclot, 49)

o. $\quad$ que viren lur senyor en terra jaure (Desclot, 49)

(31) a. c[e]ssà del dit lloc exir per tot aquel dia (CriVal, 18r, R237)

b. e con hoïren ladoncs dir que (CriVal, 23r, R247)

c. e anà-sse'n tot s[o]ls mengar figues riba la céquia (CriVal, 23v, R248)

(32) a. E ell no pot asi star tant temps (Vesp, 28)

b. tots los barons e altres vingeren aparellats al pus honradament que pogueren pasar en Jherusalem (Vesp, 23)

c. $\quad$ e amaren mes meniar lo trasaur que si (Vesp, 42)

d. fou aytentost cesar los vayls de la ciutat (Vesp, 44) 


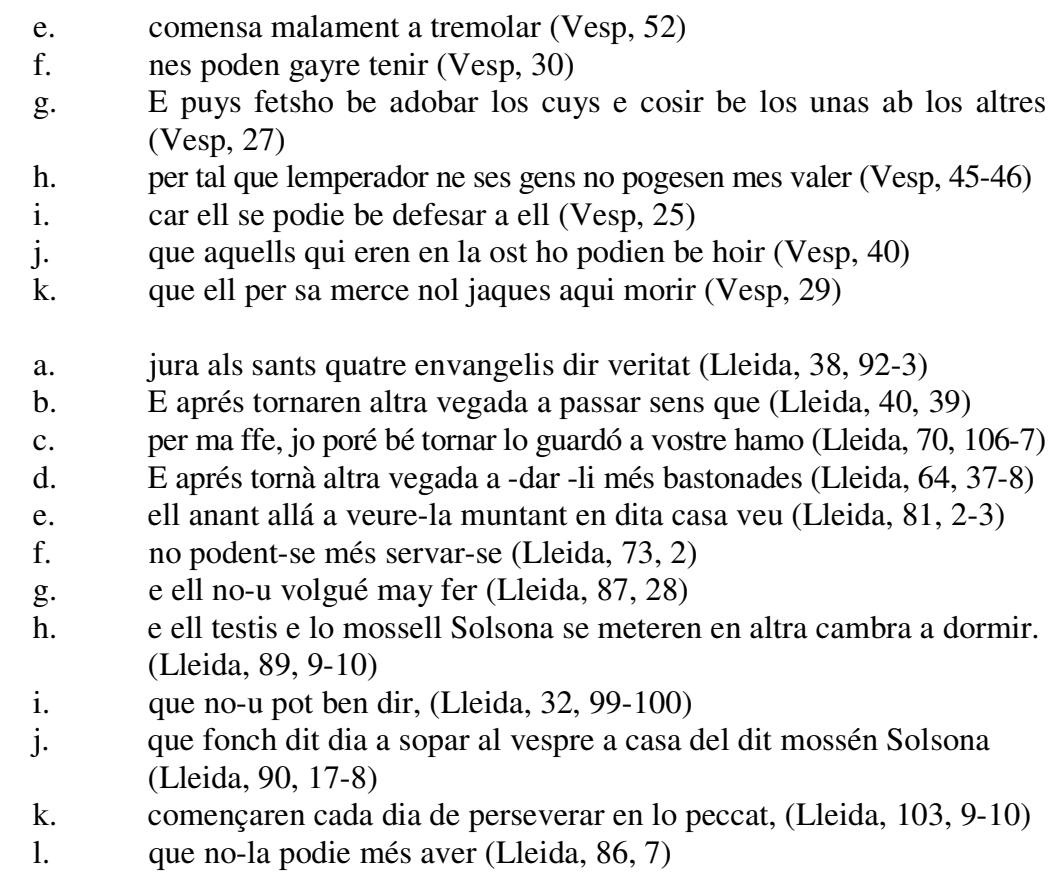

El sujeto se intercala 23 veces es decir, en el 1,9\% en todo el corpus examinado. Los casos registrados se presentan en los ejemplos del (34) al (38). Nótese que el sujeto interpolado varias veces es el sustantivo hom, que desempeña la función de sujeto indeterminado, por lo cual su presencia no tiene el mismo peso que la de un sintagma nominal léxico.

(34) Car ab aitals armes se deu primerament tot hom combatre (AveMar, $122,31-123,1)$

(35) a. II. o III. infans podrets vos partir dela (ReiHon, 55)

b. $\quad$ con ho podets vos dir que (ReiHon, 76)

(36) a. que no.ns devem tots metra en I punt de perdra (Desclot, 11)

b. noúm puix jo aventurar a tota res axí com vosaltres? (Desclot, 13)

c. que ja hi puxa negú star a prou (Desclot, 44)

(37) a. E fara hom portar aygua cascun jorn (Vesp, 27)

b. Senyer que pot hom fer daso mas que ... (Vesp, 35)

c. e not deu hom creure tu ne ton consell (Vesp, 28)

d. per ço deute hom creure meins (Vesp, 28)

e. $\quad$ e feune lemperador liurar a tots los cavalles (Vesp, 46)

f. e lexals hom anar a Deus (Vesp, 48)

g. e veuhi hom tornajar layga (Vesp, 52) 
h. e donali hom a menjar pa e aigua ben poch (Vesp, 51)

i. $\quad$ que no volgesets vos ne vostra gent asi star (Vesp, 26)

j. $\quad$ si que hanc de leyns non poch hom exir (Vesp, 35)

k. $\quad$ que ia per la siutat no pogere hom anar sino per morts (Vesp, 46)

1. que noy podie hom entrar (Vesp, 51)

(38) a. veu ell testis venir hun jove stranger (Lleida, 67, 15-6)

b. $\quad$ E veu ell ab tota sa torba ne entrar dos per la porta (Lleida, 82, 28)

c. digueren per lo cap d' aytal, mala nit los poria Déus donar que (Lleida, 26, 34-5)

d. $\quad$ los quals procehien tots en rebre un testimoni (Lleida, 92, 10-1)

e. que per quant vengué hun germà de la muller del dit Anthon a demanar-lo (Lleida, 90, 28-9)

46 veces, o sea, en el 3,9\% de las 1188 construcciones de infinitivo se ha registrado la interpolación de algún argumento interno -complemento regido- entre el verbo regente y el infinitivo dependiente. Esta proporción supera sorprendentemente a la del sujeto interpolado, lo cual no corresponde a las expectativas previas. Nótese, sin embargo, que casi la mitad de las ocurrencias de ese tipo de interpolación se ha documentado en un único texto medieval, en concreto en CriVal. Ya se ha mencionado que esta parte del corpus está constituida por diferentes textos cortos por lo general denunciaciones y sentencias tribunales- en los que se repiten monótonamente las mismas estructuras, especialmente aquella -14 veces- en la que aparece el verbo regente negar con un infinitivo dependiente cuyo sujeto funciona en la superficie como argumento interno de aquél, colocándose entre los dos verbos. La repetición de esta construcción, que se puede observar en (42a-c,) (42e) (42g-j), (421o), (42r-s), cuya estructura es semejante a la de las construcciones causativas y perceptivas causa el alto porcentaje de este tipo de interpolación, el cual seguramente no debe ser considerado normal con este verbo en la lengua corriente de la época.

ans me sent cor e força con faça a vós atorgar que la mia dona és mellor que la vostra (AveMar, 128, 25-27)

(40) a. E anch no ves festa tan poch durar (ReiHon, 60)

b. cant hoiyren al rey tan gran dol fer (ReiHon, 60)

c. qui veeren la barcha venir (ReiHon, 61)

d. ella no volch als respondre sino que (ReiHon, 62)

e. $\quad$ cant vahe al conte tan amar sa muler (ReiHon,63)

f. e veren la barqua venir sens nul govern (ReiHon, 72)

(41) a. e faré 'ls hich tots axir, (Desclot, 15)

b. e féu les cases descobrir (Desclot, 34)

c. que mantinent no.1 fes tot lancejar (45)

(42) a. neguà aqueles ésser veres (CriVal, 1r, R216)

b. neguà aqueles ésser veres (CriVal, 1r, R216bis a)

c. neguà aqueles ésser veres (CriVal, 1r, R216bis b) 
d. pronuncians aquels no ésser colpables en res del dit crim (CriVal, 1v, R217)

e. neguà aquels capítols ésser veres (CriVal, 4v, R221)

f. $\quad$ volent a mi alciure (CriVal, 7r, R224)

g. negà aquelles ésser veres (CriVal, 8r, R226)

h. negà aquelles ésser veres (CriVal, 11r, R229)

i. neguà aqueles ésser veres (CriVal, 13r, R231)

$\mathrm{j}$. neguà aqueles ésser veres (CriVal, 18r, R236)

k. axí com a $*($ des $)<$ in $>$ obedient de la seynoria e meynsprean lo manament del seynor rey e de son loch tinens no vulla lo dit deute manifestar (CriVal, 18v, R241)

1. negà aqueles ésser veres (CriVal, 20v, R243)

m. neguà aqueles ésser veres (CriVal, 22r, R245)

n. [e n]eguà aquel[les] ésser veres (CriVal, 23r, R2247)

o. neguà aqueles ésser veres (CriVal, 23r, R247)

p. diens que no y podien àls fer que (CriVal, 26r, R250)

q. que si alcun volrà aquel absent defendre a al.legar e a dir rahons d'absència suficiens (CriVal, 29r, R253)

r. E negà totes les coses contra él posades ésser veres (CriVal, 45v, R255)

s. $\quad$ neguà aqueles ésser veres (CriVal, 56r, R261)

t. On demane aquels a ela ésser condempnats per les dites injúries segons fur de València (63r, R264)

(43)

a. $\quad$ Lexem aso star (Vesp, 37)

b. mana tots sos cavalles armar (Vesp, 44)

c. Anem a lemperador cridar merce (Vesp, 24)

d. $\quad$ E puys fetsho tot scorxar e salar (Vesp, 27)

e. que misatger no devia mal pendra (Vesp, 16)

f. e cant lemperador viu lapostoli venir e ell ab ten gran profaso e ab ten gran alegria (Vesp, 49)

g. quels seus anamichs no li puxen dan tenir (Vesp, 29)

h. que non volie pus vendre (Vesp, 46)

i. $\quad$ quen podem nos alre fer que ... (Vesp, 32)

(44) a. e hoyt lo colp hóy cridar en Moyet dient (Lleida, 27, 57-8)

b. $\quad$ volents sobre lo fet de la veritat inquirir (Lleida, 34, 9-10)

c. e de continent veu lo gendre de mestre Enrich Johan Navarro pelicer, entrar darrere lo dit jove ab spassa e broquer arrencat (Lleida, 75, 8-10)

d. $\quad$ E m,s ell testis creu les coses per ell dessús denunciades ésser veres (Lleida, 80, 35-6)

e. los quals prohòmens, (...), declararen lo dit Galceran Narbó ésser caygut en ban (Lleida, 28, 90-1) 


\section{Resumen y conclusiones}

En las páginas precedentes se han presentado dos fenómenos que afectan la sintaxis de los complejos verbales de participio y de infinitivo del catalán medieval: la posibilidad de anteponer el verbo dependiente al verbo regente y la posibilidad de intercalar diversos constituyentes entre los dos verbos de las construcciones. Ambos fenómenos se observan en ambos tipos de construcciones medievales: tanto el participio como el infinitivo aparecen antepuestos al verbo flexionado que los rige; de forma parecida, tanto el participio como el infinitivo pueden ser separados por su verbo regente precedente por constituyentes intercalados.

Estas alternativas sintácticas, en la actualidad agramaticales o muy limitadas, según el testimonio de los textos catalanes medievales examinados, fueron normales, aunque su frecuencia no parece haber alcanzado - ni haberse aproximado- a la de las estructuras que hoy son las habituales. La frecuencia de la anteposición del participio dependiente es el doble de la del infinitivo. En el caso de la interpolación encontramos lo contrario: la frecuencia de la interpolación de constituyentes entre el verbo regente y el participio alcanza aproximadamente la mitad de la proporción de constituyentes intercalados entre el verbo regente y el infinitivo complemento.

La comparación de los datos de frecuencia según la época indica que la proporción de la anteposición de las formas no flexionadas al verbo regente por lo general no cambia considerablemente en el período examinado. No obstante, en los textos más tardíos -ReiHon, Vesp y Lleida- la anteposición del infinitivo desciende de modo radical, mientras que la del participio se mantiene aproximadamente al mismo nivel que en los textos anteriores. La interpolación de constituyentes entre el verbo regente y el verbo no flexionado presenta datos bastante parecidos durante todo el período examinado -aunque parece ser más habitual en las construcciones de infinitivo-, y sólo en el caso de los textos jurídicos podemos observar un ligero descenso del fenómeno en ambos tipos de construcciones. Por lo que se refiere a la comparación de los datos de frecuencia según tipos de textos, precisamente en los de carácter jurídico encontramos un porcentaje relativamente bajo -y descendente- de la interpolación especialmente en cuanto a las construcciones de participio. En los demás textos la proporción de la interpolación es algo más alta y no cambia durante el período. Los constituyentes más veces intercalados son aquellos que tienen carácter adverbial. El sujeto y los complementos regidos -cuya interpolación es agramatical o dudosa en la actualidad- también se documentan numerosas veces intercalados entre el verbo regente y el participio o el infinitivo durante todo el período examinado. Las cifras referentes a la frecuencia de estos últimos elementos deben ser tratadas con cautela, puesto que los casos de sujeto impersonal, así como los casos con complemento directo "agente" probablemente no se deberían tener en cuenta en el mismo modo que los demás casos de interpolación. 